\title{
Balloon Kyphoplasty under Three-dimensional Radiography Guidance
}

\author{
Daisuke UMEBAYASHI, ${ }^{1}$ Yu YAMAMOTO, ${ }^{1}$ Yasuhiro NAKAJIMA, ${ }^{1}$ \\ and Masahito HARA ${ }^{1}$
}

${ }^{1}$ Department of Neurosurgery, Inazawa Municipal Hospital, Inazawa, Aichi, Japan

\begin{abstract}
Percutaneous balloon kyphoplasty (PBKP) is generally performed under two-dimensional (2D) radiography guidance (lateral- and anteroposterior (A-P) views) using C-arm fluoroscopy. However, 2D images taken by single-plane or bi-plane fluoroscopy cannot provide information regarding axial views, particularly the $\mathrm{Z}$ axis. Lack of information regarding the $\mathrm{Z}$ axis prevents the creation of three-dimensional (3D) images. Currently, there has been a progress in interventional X-ray systems, and they are capable of providing 3D radiographic images using a rotational angiography mode which is used to create 3D angiographies. In this report, we described the usefulness of 3D radiography guidance. Patients treated by PBKP was designed to evaluate the efficacy of 3D radiography guidance. These patients experienced osteoporotic vertebral fractures with severe pain. We retrospectively analyzed patients who underwent PBKP from February to December 2016. All patients had a single-level vertebral fracture and underwent surgery by 2D or 3D radiography guidance. We performed 16 patients in 3D radiography guidance, and 10 patients in traditional 2D radiography guidance. This 3D radiography guided PBKP increase the amount of the polymethyl methacrylate (PMMA) injection compared with ordinary 2D method. As a result, postoperative vertebral height and alignment were significantly improved. Both groups have no complication. To confirm the final results and make PBKP more effective, 3D radiography guidance is feasible and safe for balloon kyphoplasty.
\end{abstract}

Key words: balloon kyphoplasty, 3D radiography, vertebral body fracture, osteoporosis

\section{Introduction}

Osteoporotic vertebral fractures (OVFs) are increasing in the aging populations and have become a socioeconomic problem. OVFs severely diminish the activities of daily living (ADL) in the elderly, ${ }^{1)}$ pose a major health problem to patients, and result in the shortening of patients' lives. ${ }^{2)}$ Due to the weakening of osteoporotic bone, surgery with any implants often fails, resulting in persistent back pain. Furthermore, such invasive surgery is not suitable for elderly individuals because they often present potential risks for surgery. Therefore, the advancement of less invasive surgery is warranted.

Percutaneous vertebroplasty (PVP) and the recently developed percutaneous balloon kyphoplasty (PBKP) are less invasive procedures for the treatment of OVFs. These procedures can produce immediate pain relief compared with conservative treatments. ${ }^{3,4)}$

Received December 5, 2016; Accepted March 15, 2017

Copyright $\odot 2017$ by The Japan Neurosurgical Society This work is licensed under a Creative Commons AttributionNonCommercial-NoDerivatives International License.
Wardlaw et al. reported that patients with non-operative (conservative) management had a 2.28 times greater risk for a subsequent vertebral compression fracture than patients treated with PBKP and PVP. ${ }^{5)}$ With respect to pain relief and functional status, many comparison studies have reported few differences. ${ }^{6,7)}$ However, PBKP is superior to PVP in terms of kyphosis correction, vertebral height restoration, and cement leakage prevention. ${ }^{8)}$ Therefore, PBKP has currently become a more popular procedure than PVP. In a previous report, we showed the usefulness of three-dimensional (3D) radiography guidance in PVP, which enabled the safe insertion of PVP needles. On the other hand, PBKP is a more steric procedure compared with PVP because PBKP requires the insertion of not only needles but also balloons, which create a steric structure on inflation. From this viewpoint, 3D radiography guidance is more useful in PBKP than in PVP. PBKP requires an accurate and safe technique. In particular, the appropriate maximum expansion of balloons and insertion of polymethyl methacrylate (PMMA) need a precise evaluation method to achieve effective kyphoplasty. Rotational angiography provides 3D images during 
PBKP, with relatively lower radiation exposure than computed tomography (CT) guided PBKP. However, there is no available report about $3 \mathrm{D}$ radiography guidance for BKP. Here, we demonstrate a technique for performing PBKP under 3D radiography guidance and describe its concrete advantages in the amount of PMMA injection.

\section{Methods}

\section{Patient population}

A retrospective study on patients treated with PBKP was designed to evaluate the efficacy of 3D radiography guidance. These patients experienced single level osteoporotic vertebral fractures with severe back pain. Our inclusion criteria were, fracture showing a high signal intensity in the Short-TI Inversion Recovery (STIR) magnetic resonance image (MRI) image, and compatibility between the location of the severe back pain and the level of fracture. We retrospectively reviewed patients who underwent PBKP between February and December 2016. All patients had a single-level vertebral fracture and underwent surgery with two-dimensional (2D) or 3D radiography guidance depending on the availability of the operation room. Furthermore, we evaluated the time to recovery of walking ability after BKP and performance status (PS), which may reflect the extent of back pain.

\section{Radiologic assessment}

Lateral radiographs during pre- and postoperative periods were used to assess vertebral height and vertebral kyphosis. Vertebral height was assessed according to the quantitative measurement (QM). ${ }^{9}$ Indexes of vertebral height are shown in Fig. 1A. Fractured vertebral body height was defined based on percentage compared with posterior height (P). Therefore, we assessed anterior height/posterior height (A/P) and central height/posterior height $(\mathrm{C} / \mathrm{P})$. Postoperative change in vertebral height was defined as postoperative vertebral height (\%) preoperative vertebral height (\%). Vertebral kyphosis was defined as the angle formed by the upper and lower endplates of the fractured vertebral body (Fig. 1B). Lordosis was shown as a negative value, and kyphosis was shown as a positive value. Standard statistical analysis was used for this study. We also graded the vertebral fractures from 0 to 5 using semi - quantitative measurement (SQ). ${ }^{10)}$

\section{Surgical method}

Under general anesthesia, the patients were placed in the prone position. We performed PBKP with the KYPHON BKP system (Sofamor Danek, Medtronic, TN, USA). Insertion of the transpedicular bone access
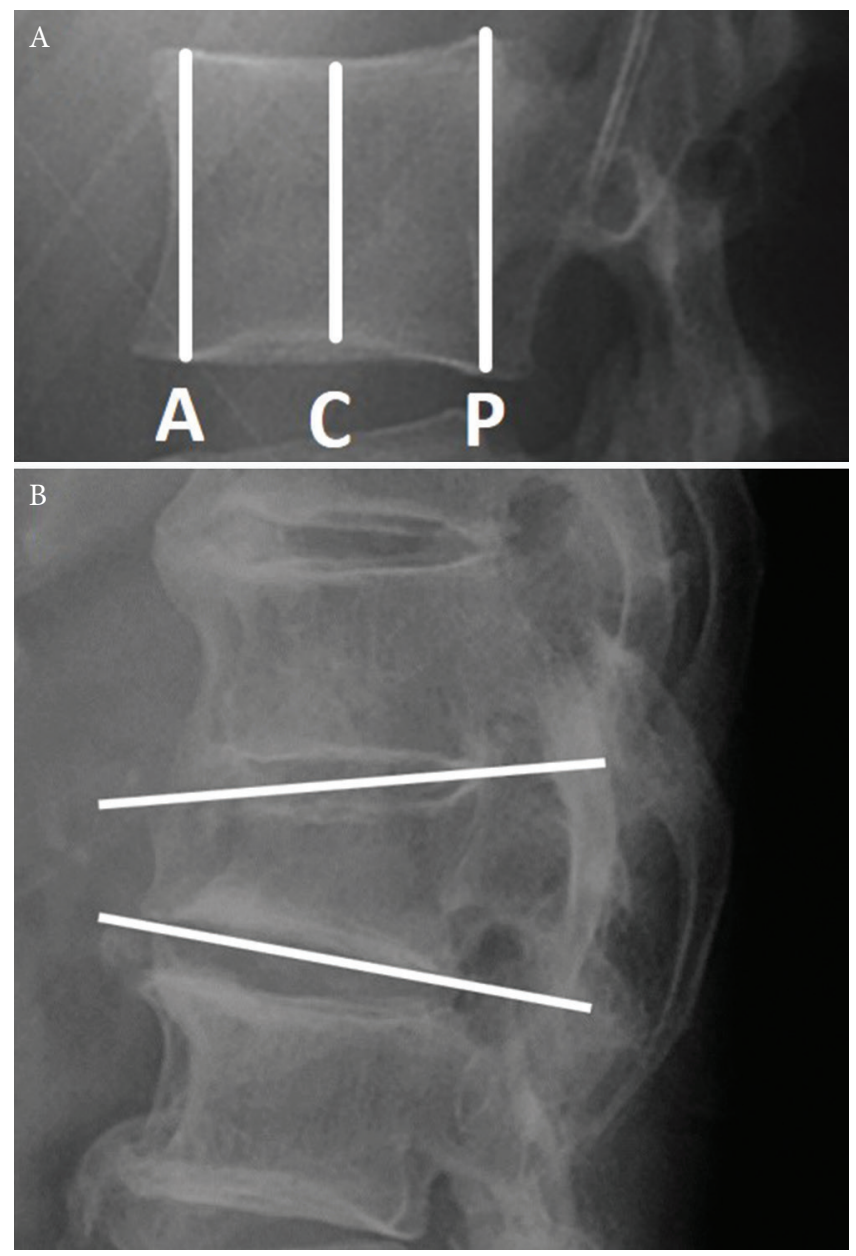

Fig. 1 (A) Radiographic measurement of vertebral height. A: Anterior height; C: central height; P: posterior height, (B) Vertebral kyphosis.

needles was performed bilaterally under 2D radiography guidance (lateral and A-P views) using combination of pair of C-arm X-ray system for 2D radiography guidance or an interventional X-ray system (AlluraClanity FD10/10; Royal Phillips Electronics, The Netherlands for 3D radiography guidance group. Subsequently, we exchanged the bone access needles for Osteo Introducers, which were hollow instruments, using blunt guidewires under 2D radiography guidance. Through the Osteo Introducers, precision drills were advanced to $3 \mathrm{~mm}$ behind the anterior wall of the vertebra under consecutive lateral fluoroscopic movie to make way for the balloons. Small balloons were then inserted through the Osteo Introducers to the position where they were expected to expand. The balloons were inflated just a little before the endpoints under 2D radiography guidance. Our endpoints were the following: achievement of the ideal vertebral height, reaching any cortexes or endplates, and reaching 400 psi balloon inflation 
pressure. Here, the first rotation scan was obtained as a $3 \mathrm{D}$ radiography image to confirm the position and the extent of expansion of the balloons in a 3D workstation (Interventional Tools Rel.9 (Basic) 3D-RA R6.4; Royal Philips Electronics, The Netherlands). Based on the rotation scan data, 3D tomography images were made. If balloon expansions were not sufficient, additional inflation was added. Further, in the case of vertebra plana, we inserted the balloon in the exact mid-position. In the case of a large cleft, we inserted the balloon just under the cleft which we were not able to see on the $2 \mathrm{D}$ radiography lateral view. If there is some uneasiness about the balloon position, we could confirm the position on a 3D tomography image. Following removal of the balloons, PMMA (KYPHON BKP OSTEO CEMENT HV) (Sofamor Danek, Medtronic, TN, USA) was gradually inserted bilaterally under 2D radiography guidance. PMMA sterile powder was mixed with methyl methacrylate monomer to produce PMMA using a KYPHON mixer (Sofamor Danek, Medtronic, USA). Using bone filler devices (nozzles and plungers), PMMA was injected under 2D radiography guidance (lateral and A-P), which was terminated when PMMA filling was achieved up to the posterior third of the vertebral body. PMMA played the role of an anchor in the posterior third of the vertebral body, preventing anterior migration of the PMMA block. Lastly, a second rotation scan was obtained to evaluate the PMMA filling, and if it was not adequate, additional PMMA filling was performed.

\section{Results}

Characteristics of patients undergoing PBKP with 2D and $3 \mathrm{D}$ radiography guidance. PMMA injection with $3 \mathrm{D}$ radiography guidance was marginally different from that with $2 \mathrm{D}$ guidance.

Between February and December 2016, 27 patients underwent PBKP. One patient was excluded due to the developed pneumothorax at the time of local anesthesia injection before PBKP. Therefore, 26 patients (9 males, 18 females, average age, $80.4 \pm 1.2$ years; range, 67-89 years) were evaluated for their population characteristics. Of the 26 patients, 16 and 10 patients underwent PBKP by 3D and 2D radiography guidance, respectively. Characteristics of 3D and 2D guidance PBKP are shown in Table 1. There was no significant difference between $3 \mathrm{D}$ and 2D guidance PBKP. However, the amount of PMMA injection during 3D radiography guidance was more than that during $2 \mathrm{D}$ radiography guidance (Table 1 , $P=0.0584$ ). There was no remarkable complication.

To determine radiological differences in the $3 \mathrm{D}$ and 2D guidance groups, 15 and 10 patients, respectively, were available for study.
Table 1 Characteristics of 3D and 2D guidance PBKP

\begin{tabular}{|c|c|c|c|}
\hline & \multicolumn{2}{|c|}{ Number of patients (\%) } & \\
\hline & 3D guidance & 2D guidance & \\
\hline Number of patients & 16 & 10 & \\
\hline Age (у.о.) & $81.6 \pm 1.3$ & $78.5 \pm 2.3$ & NS \\
\hline Sex (Male, Female) & M:5, F11 & $\mathrm{M}: 4, \mathrm{~F}: 6$ & \\
\hline \multicolumn{4}{|l|}{ Level } \\
\hline Th11 & $2(12.5 \%)$ & $1(10.0 \%)$ & \\
\hline Th12 & $1(6.3 \%)$ & $1(10.0 \%)$ & \\
\hline L1 & $7(43.8 \%)$ & $4(40.0 \%)$ & \\
\hline L2 & $3(18.8 \%)$ & $2(20.0 \%)$ & \\
\hline L3 & $2(12.5 \%)$ & $2(20.0 \%)$ & \\
\hline L4 & $1(6.3 \%)$ & $0(0.0 \%)$ & \\
\hline Complication & none & none & \\
\hline $\begin{array}{l}\text { Amount of } \\
\text { PMMA (ml) }\end{array}$ & $6.5 \pm 0.5$ & $5.1 \pm 0.3$ & $\begin{array}{c}P<0.1 \\
(P=0.0584)\end{array}$ \\
\hline Operation time (min) & $53.1 \pm 3.3$ & $55.6 \pm 3.1$ & NS \\
\hline $\begin{array}{l}\text { Time to recovery of } \\
\text { walking ability after } \\
\text { BKP (Day) }\end{array}$ & $1.8 \pm 0.3$ & $2.3 \pm 0.8$ & NS \\
\hline Preoperative PS & $2.8 \pm 0.2$ & $2.6 \pm 0.3$ & NS \\
\hline $\begin{array}{l}\text { Postoperative PS } \\
\text { (1 week after surgery) }\end{array}$ & $1.9 \pm 0.3$ & $2.1 \pm 0.2$ & NS \\
\hline $\begin{array}{l}\text { Postoperative PS } \\
\text { (1 month after surgery) }\end{array}$ & $1.4 \pm 0.3$ & $1.6 \pm 0.2$ & NS \\
\hline Improvement of PS & $1.4 \pm 0.2$ & $1.0 \pm 0.3$ & NS \\
\hline Total 26 patients & & & \\
\hline
\end{tabular}

Postoperative vertebral morphology was better visualized in the $3 \mathrm{D}$ than in the $2 \mathrm{D}$ radiography guidance groups

Vertebral height was defined as the percentage of its posterior height (A/P, C/P). Preoperative reduction in height of the vertebra was not significantly different between the 3D and 2D radiography guidance groups (Table 2). However, postoperative height of the vertebra $(\mathrm{A} / \mathrm{P}, \mathrm{C} / \mathrm{P})$ was significantly taller in the $3 \mathrm{D}$ than in the $2 \mathrm{D}$ radiography guidance groups (Table 2; A/P, $P=0.00564, \mathrm{C} / \mathrm{P}, P=0.00928$ ), and SQ grade was significantly shorter in the $3 \mathrm{D}$ than in the 2D groups (Table 2, $P=0.01630$ ). Furthermore, vertebral kyphosis in the 3D group was also significantly lower than that in the 2D postoperative radiography group (Table 2, $P=0.00198$ ).

\section{D radiography provides axial view vertebral images with low radiation exposure}

$2 \mathrm{D}$ radiography guidance, which consists of lateral and A-P fluoroscopy images, cannot provide information regarding axial views, particularly the $\mathrm{Z}$ axis. However, 3D images taken in the rotational 
Table 2 Differences in pre- and postoperative radiography between $3 \mathrm{D}$ and $2 \mathrm{D}$ guidance $\mathrm{PBKP}$

$3 \mathrm{D}$ guidance $2 \mathrm{D}$ guidance

\begin{tabular}{lrrc}
\hline $\begin{array}{l}\text { Preoperative } \\
\text { reduction in height } \\
\text { of vertebra }\end{array}$ & & & \\
A/P (\%) & $78.5 \pm 7.9$ & $63.7 \pm 3.8$ & NS \\
C/P (\%) & $56.4 \pm 4.0$ & $58.5 \pm 3.6$ & NS \\
SQ & $2.4 \pm 0.5$ & $2.4 \pm 0.7$ & NS \\
Vertebral kyphosis & $10.1 \pm 2.5$ & $16.0 \pm 1.8$ & NS \\
$\begin{array}{l}\text { Postoperative } \\
\text { height of vertebra }\end{array}$ & & & \\
$\begin{array}{l}\text { A/P (\%) } \\
\text { C/P (\%) }\end{array}$ & $91.4 \pm 4.6$ & $70.6 \pm 4.6$ & $P<0.05$ \\
$\begin{array}{l}\text { SQ } \\
\text { Vertebral kyphosis }\end{array}$ & $3.7 \pm 1.6$ & $12.2 \pm 1.8$ & $P<0.05$ \\
$\begin{array}{l}\text { Postoperative } \\
\text { change in the }\end{array}$ & & & \\
vertebral height & & & \\
$\begin{array}{l}\text { A/P (\%) } \\
\text { C/P (\%) }\end{array}$ & $12.8 \pm 4.7$ & $6.9 \pm 3.4$ & NS \\
$\begin{array}{l}\text { Postoperative } \\
\text { change in kyphosis }\end{array}$ & $19.7 \pm 2.8$ & $3.4 \pm 3.5$ & $P<0.05$ \\
$\begin{array}{l}\text { Improvement of } \\
\text { kyphosis angle }\left({ }^{\circ}\right)\end{array}$ & $6.4 \pm 1.6$ & $3.8 \pm 1.4$ & NS \\
$\begin{array}{l}\text { Number of } \\
\text { patients }\end{array}$ & & & \\
\hline
\end{tabular}

angiography mode (Figs. 2 and 3 ) are able to provide axial view images in the same manner as tomography images (Fig. 4A). Moreover, these 3D images provide coronal and sagittal views (Figs. 4B and C). A 3D image taken in the rotational angiography mode requires only $23 \mathrm{mGy} / \mathrm{mal}$, which is almost identical to the requirement of a CT scan (18 $\mathrm{mGy} / \mathrm{mal}$ ).

The extent of balloon inflation can be easily evaluated by $3 \mathrm{D}$ radiography images.

As a result, the maximum inflation of the balloon increases the amount of PMMA injection.

If a structure is symmetrical and the endplates are flat and parallel, 2D radiography images are sufficient for its evaluation. However, a fractured vertebral body has a complex asymmetrical structure with unparalleled endplates. Furthermore, in the round structure of the vertebral body, the four corners and the tips of the antero- and posterolateral points are always in the dead angle areas in 2D radiography guidance because neither lateral nor coronal images describe the surface lines of the anterolateral portion of a vertebra (Fig. 5). However, 3D radiography images

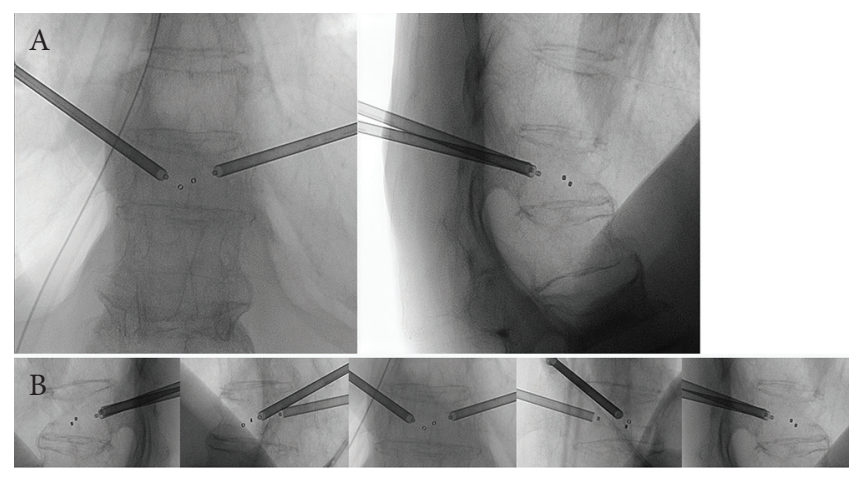

Fig. 2 (A) Lateral and A-P view. (B) 3D images taken using the rotational angiography mode. Balloons were inserted bilaterally via the Osteo Introducer system.

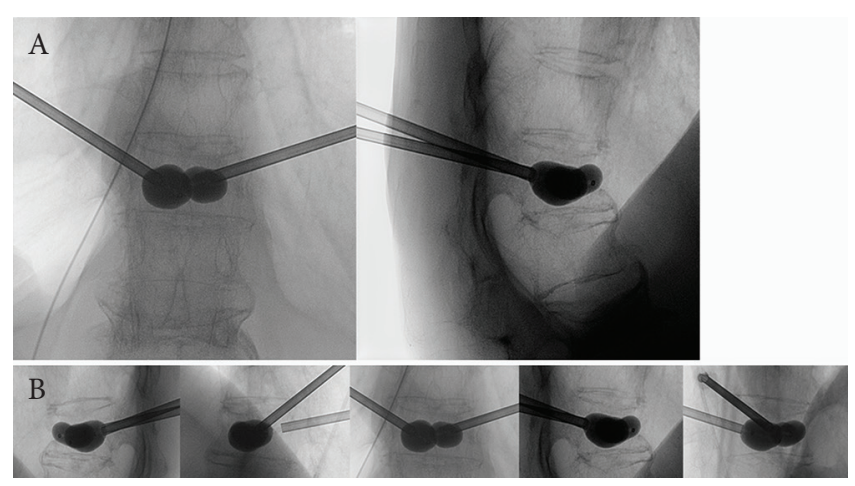

Fig. 3 (A) Lateral and A-P view. (B) 3D images taken using the rotational angiography mode. Balloons were inflated bilaterally near the endpoint. The right balloon was advanced compared with the left.

facilitate the recognition of all surface lines of the vertebra (Fig. 4). If the balloon expansions are not sufficient, additional inflation can be added easier during 3D radiography guidance than during 2D radiography guidance. This maximum inflation of the balloon resulted in increase of PMMA injection. On the other hand, 3D radiography guidance also facilitate the recognition of all surface lines of the injected PMMA. However account of the temporal restriction of the PMMA hardens, it is hard to inject additional PMMA.

\section{D radiographic images distinguish the right balloon from the left}

$3 \mathrm{D}$ radiographic images also made it easy to distinguish which balloon was anterior to the other, in the case that the contrast agent of the balloons concealed the marker and catheters following inflation (Fig. 3). In such cases, we often find it difficult to determine which balloon should receive added inflation; 3D radiography images easily solve the problem. 

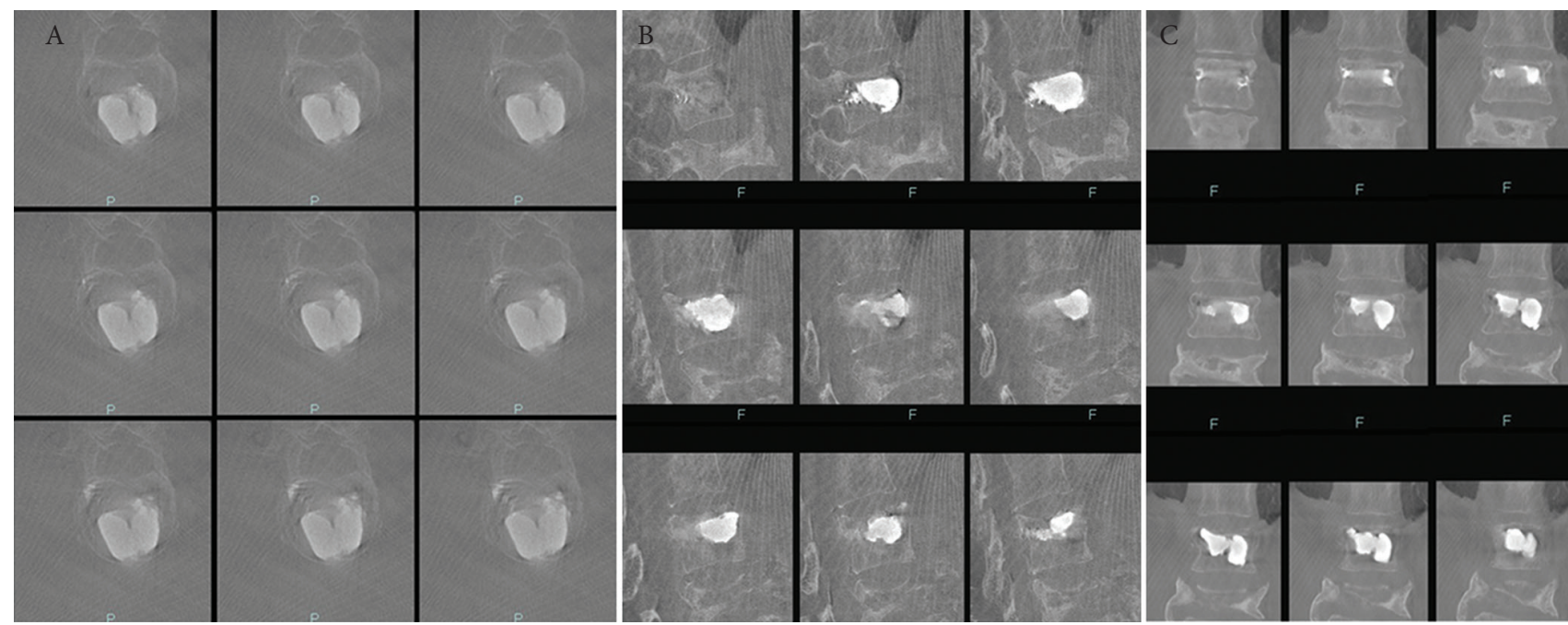

Fig. 4 Axial view images similar to tomography images (A). Sagittal (B), and coronal (C) view images.

\begin{abstract}
Prevention of PMMA leakage and confirmation of the appropriate extent of the posterior anchor

From 2D images, it is sometimes difficult to distinguish PMMA leakage at the tip of the vertebrae. 3D images help to distinguish PMMA leakage by looking from multi-angle views, in particular, tomography images confirm the leakage (Fig. 4).
\end{abstract}

\section{Discussion}

3D radiography aids the insertion of devices. Expert spine surgeons who are familiar with the insertion of pedicle screws can easily insert bone access needles using only 2D radiography. In contrast, it is difficult for a non-expert surgeon to correctly insert such devices via pedicles. Moreover, a failed insertion could result in severe complications, such as nerve injury, surrounding organ injury, and PMMA leakage. $3 \mathrm{D}$ radiography guidance allows even a non-expert to correctly and safely approach the vertebral body percutaneously via pedicles. ${ }^{11,12)}$

The present study shows that BPKP was performed under 3D radiography guidance, to make kyphoplasty more effective. The latest interventional X-ray systems have a rotational angiography mode for the creation of 3D angiographies. This can also be used to create 3D images in bones. Therefore, it enables to create 3D images during PBKP, with a relatively low radiation exposure. PBKP is usually performed under 2D radiography guidance using single- or bi-plane fluoroscopy or CT guidance. ${ }^{5,13,14)}$ Single- or bi-plane fluoroscopy images cannot provide information regarding the $\mathrm{Z}$ axis. 2D images could be accurate enough to determine the appropriate trajectory if an expert spine surgeon were to perform PBKP. However, if the deformity of the fractured vertebra
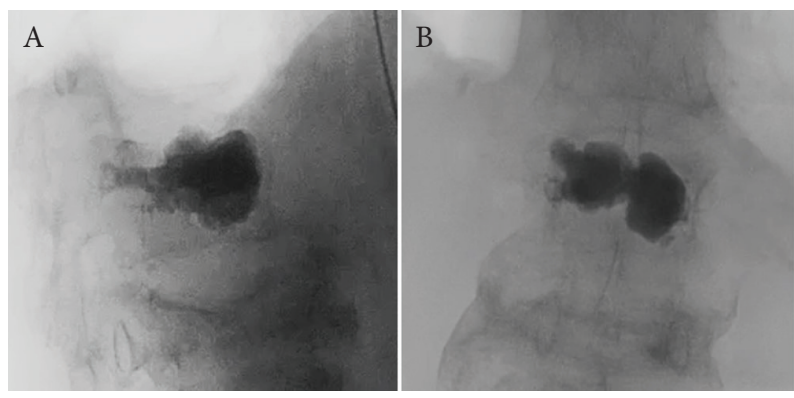

Fig. 5 In such a round structure of the vertebral body, the four corners and the tips of the antero- and posterolateral points are always in the dead angle areas in 2D radiography guidance because neither lateral (A) nor coronal (B) images describe the surface lines of the anterolateral portion of a vertebra (Fig. 4).

is severe, it is difficult to confirm the accurate final results from 2D images alone. Therefore, many surgeons perform a CT scan after surgery to follow up and confirm the results. Postoperative CT scans also increase radiation exposure.

Some surgeons perform PBKP under CT guidance. However, CT-guided PBKP always requires fluoroscopy because PBKP requires evaluation of the balloon expansion height. Therefore, CT-guided PBKP requires movement of a portable fluoroscopy unit, which requires a spacious CT room and suspension of routine daily work.

Furthermore, interventional X-ray systems in the angiography room improve the image intensifier because that of the C-arm fluoroscopy equipment is relatively low image intensifier.

Radiation exposure during PBKP is approximately $76 \mathrm{mGy}$ for 3D radiography [rotational scan $23 \mathrm{mGy} /$ mal $\times 2$ (ballooning, PMMA insertion) + fluoroscopy 
$15 \mathrm{mGy} / \mathrm{min} \times 2 \mathrm{~min}]$. In contrast, the radiation exposure is $90 \mathrm{mGy}$ for CT guidance [CT $18 \mathrm{mGy} / \mathrm{mal}$ $\times 5$ (bone access needle, Osteo Introducer, precision drill, ballooning, PMMA insertion)]. In addition, under conventional fluoroscopy guidance, many surgeons perform a CT scan (18 mGy) following surgery. As a result, PBKP under 3D radiography guidance decreases the radiation exposure for not only patients but also surgeons.

$3 \mathrm{D}$ radiography guidance increased the amount of PMMA injection. However, if the PMMA injections are not sufficient, additional injections are difficult due to the temporal restriction of the hardened PMMA. Increased of PMMA injection may be due to the increased balloon expansions because additional inflation of the balloon can be added more easily during $3 \mathrm{D}$ rather than $2 \mathrm{D}$ radiography guidance. This increased amount of PMMA injection may affect postoperative vertebral morphology improvement during 3D radiography guidance.

Operation times during 3D and 2D radiography guidance were not significantly different despite $3 \mathrm{D}$ radiography guidance requiring additional time for three times rotational angiography. Threedimensional radiography guidance using the bi-plane angiography system can provide A-P and lateral view images at the same time in the same display. On the other hand, traditional 2D radiography guidance by a combination of two pieces of the C-arm fluoroscopy system is not able to provide $\mathrm{A} / \mathrm{P}$ and lateral view images in the same display. Therefore, bi-plane angiography system could shorten operation time.

In conclusion, PBKP under 3D radiography guidance has several advantages over conventional methods. It overcomes the disadvantage of CT-guided PBKP, which is the lack of real-time sagittal and coronal images. The interventional X-ray system we mentioned here for 3D radiography guidance is a commonly used rotational angiography system, which neurosurgeons generally use for intracranial angiography. The key point of this technical note is that all neurosurgeons are able to perform BKP under $3 \mathrm{D}$ radiography guidance just by the application of such a general angiography system which many institutions already have.

\section{Acknowledgment}

We thank Y. Tsuzuki and M. Koshizuka for their radiographic technical assistance, and K. Ando, S. Kakehi, M. Yokohashi and S. Oyama for the set-up and arrangement of the operating room.

\section{Conflicts of Interest Disclosure}

The authors declare no competing financial interests.

\section{References}

1) Cockerill W, Lunt M, Silman AJ, et al.: Health-related quality of life and radiographic vertebral fracture. Osteoporos Int 15: 113-119, 2004

2) Johnell O, Kanis JA, Odén A, et al.: Mortality after osteoporotic fractures. Osteoporos Int 15: 38-42, 2004

3) Klazen CA, Lohle PN, de Vries J, et al.: Vertebroplasty versus conservative treatment in acute osteoporotic vertebral compression fractures (Vertos II): an openlabel randomised trial. Lancet 376: 1085-1092, 2010

4) Wardlaw D, Cummings SR, Van Meirhaeghe J, et al.: Efficacy and safety of balloon kyphoplasty compared with non-surgical care for vertebral compression fracture (FREE): a randomised controlled trial. Lancet 373: 1016-1024, 2009

5) Faloon MJ, Ruoff M, Deshpande C, et al.: Risk factors associated with adjacent and remote- level pathologic vertebral compression fracture following balloon kyphoplasty: 2-year follow-up comparison versus conservative treatment. $J$ Long Term Eff Med Implants 25: 313-319, 2015

6) Diamond TH, Clark WA: Percutaneous vertebroplasty: a novel treatment for acute vertebral fractures. Med J Aust 174: 398-400, 2001

7) Garfin SR, Yuan HA, Reiley MA: New technologies in spine: kyphoplasty and vertebroplasty for the treatment of painful osteoporotic compression fractures. Spine 26: 1511-1515, 2001

8) Kim KH, Kuh SU, Chin DK, et al.: Kyphoplasty versus vertebroplasty: restoration of vertebral body height and correction of kyphotic deformity with special attention to the shape of the fractured vertebrae. J Spinal Disord Tech 25: 338-344, 2012

9) Kiel D: Assessing vertebral fractures. National osteoporosis foundation working group on vertebral fractures. J Bone Miner Res 10: 518-523, 1995

10) Genant HK, Wu CY, van Kuijk C, Nevitt MC: Vertebral fracture assessment using a semiquantitative technique. J Bone Miner Res 8: 1137-1148, 1993

11) Tenjin H, Mandai A, Umebayashi D, et al.: Percutaneous vertebroplasty under three-dimensional radiography guidance. Technical note. Neurol Med Chir (Tokyo) 49: 179-183, 2009

12) Tam AL, Mohamed A, Pfister M, et al.: C-arm cone beam computed tomography needle path overlay for fluoroscopic guided vertebroplasty. Spine 35: 1095-1099, 2010

13) Liu JT, Li CS, Chang CS, Liao WJ: Long-term follow-up study of osteoporotic vertebral compression fracture treated using balloon kyphoplasty 
and vertebroplasty. J Neurosurg Spine 23: 94-98, 2015

14) Amoretti N, Marcy PY, Lesbats-Jacquot V, et al.: Combined CT and fluoroscopic guidance of balloon kyphoplasty versus fluoroscopy-only procedures. Skeletal Radiol 38: 703-707, 2009
Address reprint requests to: Daisuke Umebayashi, MD, $\mathrm{PhD}$, Department of Neurosurgery, Inazawa Municipal Hospital, 100 Numa, Nagatsuka-cho, Inazawa, Aichi 492-8510, Japan.

e-mail: umebayad@gmail.com 